\title{
Genetic variation in recombination in Drosophila. I. Responses to selection and preliminary genetic analysis
}

\author{
Brian Charlesworth and \\ Deborah Charlesworth
}

\author{
School of Biological Sciences, University of Sussex, \\ Brighton BN1 9QG, UK.*
}

\begin{abstract}
Artificial selection was practised for increased and decreased rates of recombination between two pairs of 3rd chromosome genes $G l, S b$ and $S b, S e r$, in female Drosophila melanogaster. These genes had been placed on a genetic background derived largely from a natural population (IV), prior to the start of selection. One line out of sixteen showed a significant response to selection; recombination between $G l$ and $S b$ was increased from 13 per cent to 22 per cent. There was no evidence for directional dominance in the $\mathrm{F} 1$ between the $G l-S b$ high recombination stock and the unselected control, but there was a small $(\approx 1.5$ per cent) but significant reciprocal difference. Backcross experiments showed that this was due to a grandmaternal effect of the nuclear genotype, probably controlled by genes on chromosome 3. These experiments also demonstrated that chromosomes 2 and 3 contributed significantly to the difference between the stocks; there was suggestive but not conclusive evidence for an $\mathrm{X}$ chromosome effect. Salivary chromosome studies ruled out any role of cytologically detectable chromosome rearrangements in these effects.
\end{abstract}

\section{INTRODUCTION}

There is now a large literature on the genetic control of recombination in eukaryotes, reviewed by Baker et al. (1976), Lindsley and Sandler (1977), Catcheside (1977), Stahl (1979), and Whitehouse (1982). Much of this information has been obtained from the study of mutations with drastic effects on recombination frequencies, which have been isolated by screening procedures. While the properties of such mutations are of great interest in relation to the mechanisms of genetic control of recombination, they are not necessarily representative of the properties of genes that are important in adjusting recombination rates in natural populations. Evidence concerning the nature of variation in recombination rates due to genes of relatively minor effect, segregating in natural populations, is of much greater interest from this point of view (Maynard Smith, 1978). Unexpected features of the genetic control of recombination may be uncovered by the study of natural variation (Catcheside, 1977, Chap. 4); examples of these will be described in the third paper in this series.

A considerable number of studies of quantitative variability in recombination rates have been

* Present address: Department of Biology, University of Chicago, 1103 E. 57th St., Chicago, IL 60637, U.S.A. carried out, mostly using artificial selection to produce stocks with increased or reduced recombination rates; these have been reviewed by Maynard Smith (1978, Chapter 5) and Turner (1979). (More recent studies include those by Ebinuma and Yoshitake (1981) on the silkworm, and Flexon and Rodell (1982) on Drosophila melanogaster). Particularly informative studies of $D$. melanogaster have been carried out by Chinnici $(1971 a, b)$ and Kidwell $(1972 a, b)$, but there is still a lack of detailed information concerning the properties of genes involved in such variation, particularly with respect to their numbers and chromosomal locations and their specificity of action in relation to which sections of the genome they affect. Furthermore, earlier workers have not attempted to ensure that the genetic background of the stocks used to study variation is representative of that of a natural population. This hampers inferences about the nature of genetic variation in recombination frequencies in wild populations.

The work described in this paper and its companions is intended to obtain information of this kind, using a laboratory population of $D$. melanogaster with a genetic make-up representative of a natural population as the source material. Selection for increased and decreased recombination between two pairs of 3 rd chromosome genes ( $G l$ and $S b, S b$ and $S e r$ ) was practised for up to 
18 generations. A stock with an increased frequency of recombination between $G l$ and $S b$ was obtained, and subjected to a variety of genetic experiments. This paper describes the responses to selection, and the results of some preliminary genetic analyses of the high recombination stock. The next paper (Charlesworth and Charlesworth, 1985 ) will describe the results of measurements of the effects on $G l-S b$ recombination of individual chromosomes from the high recombination stock, and an attempt to localise some of the genes uncovered in these experiments. It also contains a discussion of the results presented in both papers. The third paper will be concerned with the characterisation of various properties of the high recombination genes, particularly their regional specificity of action.

\section{MATERIALS AND METHODS}

\section{Culture methods}

Flies were reared in a constant temperature room at $25 \pm 1^{\circ} \mathrm{C}$, in $\frac{1}{3}$ pint milk bottles or $3^{\prime \prime} \times 1^{\prime \prime}$ vials containing Lewis' medium. In the experiments described in this paper, all measurements of recombination were carried out using flies reared in bottles: matings were set up in vials and transferred to bottles 24 hours later. The selection lines were run using 10 pairs of flies per bottle, and the emergence over 5 days was used to score recombination frequencies. The later experiments on recombination also usually used 10 -pair matings, but emergence over 8 days was scored (not necessarily daily). The virgin females used to set up cultures were collected over the first three days of emergence, and were between 2-5 day's old when the bottles were set up. Flies were handled using $\mathrm{CO}_{2}$ anaesthesia.

\section{Genetic stocks used}

\section{The wild-type background}

The genetic background of the selection lines was derived largely from a stock which was constructed in the following way. About 200 wild flies of each sex were obtained in August 1975 from Dr P. T. Ives of Amherst, Massachusetts, who captured them from a large natural population at Amherst. These were used to found a stock which was maintained by mass transfer, using 10 bottles with about 40 pairs per bottle in each generation. Each new bottle was founded from a pair of new bottles of the preceeding generation, so that the set of 10 bottles constituted one breeding population. In this way, a stock with a level of genetic variability comparable to that of the original population was obtained (Rose and Charlesworth, 1981).

In October 1976, salivary chromosomes from a sample of larvae were analysed using the method of Nicoletti (1959). As expected, the population was polymorphic for inversions of chromosomes 2 and 3 . Since the presence of inversions would have seriously interfered with selection for genic modifiers of recombination, an inversion-free stock was constructed; 50 lines were founded and maintained by single-pair matings. Each line was then screened for inversion heterozygosity, and classified as inversion-free if at least 50 larvae were scored and found to be structural homozygotes. 21 such lines were obtained, and checked for homozygosity for the standard gene sequence by crossing to Oregon-R. They were then used to found an inversion-free stock (IV) by allowing them to mate at random. This stock was then maintained in a similar way to the original stock, which was discarded. Starch-gel electrophoresis for the enzymes ADH, PGM and $\alpha$-GPD was carried out in order to check that the IV stock had the level of genetic variability expected for a natural population.

\section{Genetic markers used in the selection lines}

The chromosome 3 dominant markers (with recessive lethal effects) Glued $(G l)$, Stubble $(S b)$, and Serrate (Ser), located respectively at map positions $41 \cdot 0,58 \cdot 2$ and 92.0 (Lindsley and Grell, 1968), were used in the selection experiments. These were obtained from balanced stocks maintained at the stock centre at the California Institute of Technology, and were transferred onto a background of IV lst (X) and 2nd chromosomes using the 2nd chromosome balancer $S M 1$. In order to ensure that the 3 rd chromosomes used in the selection lines were largely derived from the IV stock, the resulting lines were backcrossed to IV males for 12 generations, using females heterozygous for the markers. Four bottles of 10-pair matings were used in each generation for each marker stock. A mean of 8.3 map units on each side of the marker gene is expected to be derived from the background of the original marker stock at the start of the selection lines (Crow and Kimura, 1970, p. 95); the remainder of the genome is expected to come from IV. A set of marker strains substantially identical to IV was thus obtained, and used as the base for the selection experiments. The strains were maintained by further backcrosses to IV. 


\section{Balancers and other markers used}

In order to construct the isogenic stocks described below, the multiply-inverted chromosomes FM7, SM1 and TM6 (balancing the 1st, 2nd and 3 rd chromosomes, respectively) were employed. Details of the genetic constitutions of these chromosomes are given in the Computerised Stock List 3 (Lindsley and Zimm, 1982). FM7 was maintained homozygous, and $S M 1$ and $T M 6$ were maintained as a balanced stock $S M 1 / P m$; $T M 6 / S b$. A multiple stock $F M 7 ; S M 1 / P m$ was also constructed by us and maintained separately. A stock homozygous for the recessive markers $c n$ $b w$; ri e was also used in some of the experiments; both this and the $S M 1 / P m ; T M 6 / S b$ stock were provided by Dr C. H. Langley of NIEHS, N. Carolina.

\section{High Lines (H)}

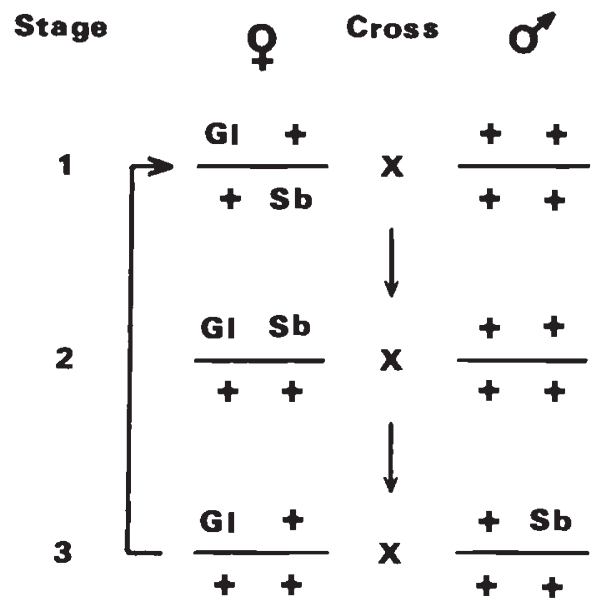

selection lines are shown in fig. 1, for the case of $G l$ and $S b$. There was no selection every 3 rd generation in the $\mathrm{H}$ lines, but selection was practised each generation in the $\mathrm{L}$ lines. The nature of the crosses used in the selection lines enabled the recombination fraction to be estimated from the phenotype frequencies observed in stages 1 and 2 of the $\mathrm{H}$ lines, and each generation of the $\mathrm{L}$ lines. Unfortunately, the marker genes have effects on viability, so that the recombination fractions estimated in this way are not strictly compatible with standard values. For this reason, control crosses were set up using the unselected markers in generations $4,10,13$ and 18 of the $\mathrm{H}$ lines, and 3, 9, 12 and 17 of the L lines; two bottles of each type of control and two bottles of each selection line were scored in these experiments.

Low Lines (L)

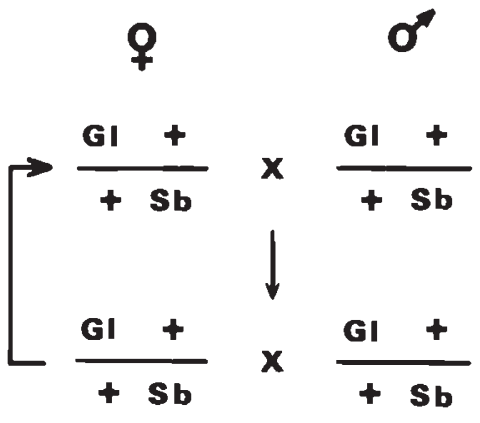

Figure 1 The scheme of selection for increased $(\mathrm{H})$ and reduced ( $\mathrm{L}$ ) recombination. Recombinant progeny only are used for breeding in stages 1 and 2 of the $\mathrm{H}$ lines; non-recombinants are used in each generation of the $\mathrm{L}$ lines.

\section{SELECTION LINES}

\section{Selection procedure}

Selection was practised for both increased and decreased recombination between $G l$ and $S b$, and between $S b$ and $S e r$. Four replicates of each selection line were run; two of these were founded by one reciprocal cross between the marker strains involved, and two from the other (e.g., $q S b \times G l$ o and $\$ G l \times S b \delta$ respectively). 16 lines in total were thus maintained; 20 pairs distributed between 2 bottles were used for each selection line. The breeding procedures for the high $(\mathrm{H})$ and low $(\mathrm{L})$
The selection lines were initiated in December 1977 in the Laboratory of Genetics, National Institute of Environmental Health Sciences, North Carolina, and were moved to the University of Sussex in late September, 1978. The move corresponded to generation 15 , which was at stage 3 for the $\mathrm{H}$ lines; the $\mathrm{L}$ lines could not be scored in this generation, although selection was practised. Selection was suspended at generation 18 for the surviving $\mathrm{H}$ lines, and at generation 17 for the $\mathrm{L}$ lines. These lines were maintained in mass culture by selecting 40 non-virgin marker females from each line every generation, which were used to set up 4 bottles of stock. 


\section{Selection responses}

\section{Measurements during the course of selection}

The estimated recombination frequencies in the selection experiments (using pooled data from the two replicate bottles of each line) for the generations when control measurements were carried out are shown in table 1 . It was apparent after a few generations that the $\mathrm{H}$ lines for $\mathrm{Sb}$-Ser were not responding to selection, and they were discarded after generation 13 . The line L Sb $\times$ Ser I was contaminated in generation 2 and was restarted from L Sb $\times$ Ser 2 . H Ser $\times$ Sb 2 was contaminated both coupling and repulsion doubly heterozygous females, and mating them to wild-type males derived from the IV stock e.g.,

$$
\begin{array}{cc}
\text { Coupling } & \text { Repulsion } \\
\uparrow \frac{G l S b}{++} \times \frac{++}{++} \delta & \wp \frac{G l+}{+S b} \times \frac{++}{++} \delta
\end{array}
$$

These crosses were carried out for the remaining $\mathrm{H}$ and $\mathrm{L}$ lines, at the same time as control crosses involving the unselected markers, and provide a means of estimating recombination frequencies in the presence of segregation anomalies due

\begin{tabular}{|c|c|c|c|c|c|c|c|c|c|c|}
\hline \multicolumn{11}{|l|}{ L Lines } \\
\hline \multirow[t]{2}{*}{ Generation } & \multicolumn{2}{|l|}{$S b \times G l$} & \multicolumn{2}{|l|}{$G l \times S b$} & \multirow[t]{2}{*}{ Control } & \multicolumn{2}{|c|}{$S b \times S e r$} & \multicolumn{2}{|c|}{$S e r \times S b$} & \multirow[t]{2}{*}{ Control } \\
\hline & 1 & 2 & 1 & 2 & & 1 & 2 & 1 & 2 & \\
\hline 9 & 0.075 & 0.080 & $0 \cdot 069$ & 0.097 & $0 \cdot 123$ & 0.388 & 0.282 & 0.406 & 0.385 & 0.525 \\
\hline 12 & $0.076^{*}$ & $0.067^{*}$ & 0.092 & $0 \cdot 101$ & 0.098 & 0.439 & 0.315 & 0.411 & 0.345 & 0.470 \\
\hline 17 & 0.076 & $0.067^{*}$ & $0 \cdot 140$ & $0 \cdot 107$ & $0 \cdot 140$ & 0.378 & 0.252 & $0 \cdot 382$ & 0.355 & 0.461 \\
\hline \multicolumn{11}{|l|}{$\mathrm{H}$ Lines } \\
\hline \multirow[t]{2}{*}{ Generation } & \multicolumn{2}{|l|}{$S b \times G l$} & \multicolumn{2}{|l|}{$G l \times S b$} & \multirow[t]{2}{*}{ Control } & \multicolumn{2}{|c|}{$S b \times S e r$} & \multicolumn{2}{|c|}{$S e r \times S b$} & Control \\
\hline & 1 & 2 & 1 & 2 & & 1 & 2 & 1 & 2 & \\
\hline 4 & $0 \cdot 109$ & $0 \cdot 161$ & $0 \cdot 160$ & 0.089 & 0.085 & 0.400 & $0 \cdot 381$ & 0.461 & 0.417 & 0.375 \\
\hline 10 & 0.117 & 0.136 & 0.110 & 0.145 & 0.097 & 0.341 & 0.367 & $0 \cdot 398$ & $0 \cdot 363$ & 0.385 \\
\hline 13 & $0 \cdot 166$ & $0 \cdot 122$ & 0.134 & 0.141 & 0.113 & $0 \cdot 340$ & 0.362 & 0.412 & 0.419 & 0.371 \\
\hline 18 & $0 \cdot 171$ & 0.139 & 0.155 & $0.240 \dagger$ & 0.121 & - & - & - & - & - \\
\hline
\end{tabular}
to viability effects of the markers (Bailey, 1961).

Table 1 Progress of the $\mathrm{L}$ and $\mathrm{H}$ selection lines. Recombination frequencies (from numbers pooled over replicate cultures) are shown, for generations when controls were measured

$\mathrm{L} S b \times G l 1$ was contaminated in gen. 2 and restarted from $\mathrm{L} S b \times G l 2 ; \mathrm{L} G l \times S b 2$ was found to contain an inversion of chromosome 2 in gen. 11 and was restarted from $\mathrm{L} S b \times G l 1 ; \mathrm{H} \mathrm{Ser} \times S b 2$ was contaminated in gen. 5 and restarted from H $S e r \times S b 1$. Asterisks $\left(^{*}\right)$ indicate that the lines differ from the control at the $\mathrm{p}<0.05$ level by a $t$-test. Each recombination fraction is based upon about 1000 flies.

$\doteqdot$ This value has an unusually high variance.

in generation 5 and restarted from $\mathrm{H}$ Ser $\times S b 1$. The selection lines were checked for chromosome rearrangements in generation 11 , by examination of salivary chromosomes of 20 larvae from each line. $\mathrm{L} G l \times S b 2$ was found to be segregating for an inversion of chromosome 2 , and was replaced by flies from $\mathrm{L} S b \times G l$ l. The lines remaining after the suspension of selection were rechecked for chromosome rearrangements about 4 generations after relaxation of selection, and were found to be karyotypically normal. Any differences from the controls must therefore be of genic or cytoplasmic origin.

\section{Tests of the selected lines}

In order to measure the selected lines more thoroughly, "balanced" measurements of recombination frequencies were carried out by constructing
Furthermore, the outcross to IV minimises any viability effects due to associations of the marker genes with recessive, detrimental factors within the selected lines (see below).

Three to five (usually four) bottles were set up for each cross; the controls and selection lines were assayed at the same time, but the three different classes of selection line shown in table 2 were assayed in different weeks. The values shown in table 2 are the unweighted means and standard errors of the recombination frequencies of each cross. $t$-tests were carried out using Fisher's angular transform; no significant differences between the coupling and repulsion crosses were detected, so that comparisons between the selected lines and their controls were based on pooled coupling and repulsion values.

The only selection line to differ significantly from the control was $\mathrm{H} S b \times G l$ l, although several 
Table 2 Balanced tests of the selected lines and controls

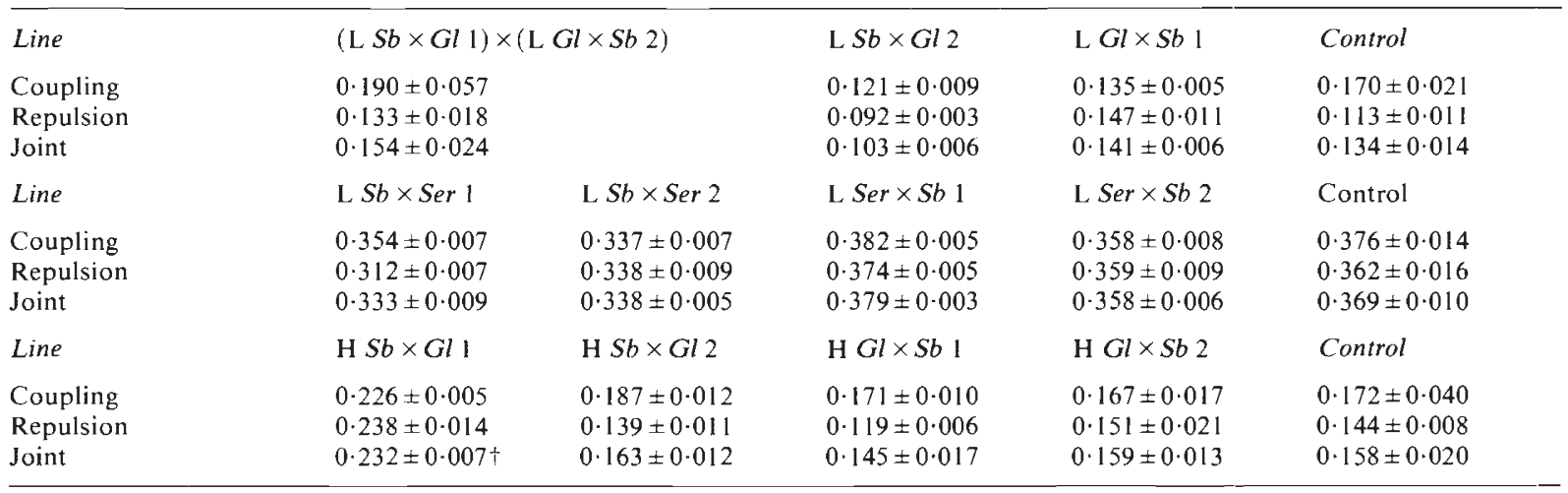

Each coupling or repulsion value is based on about 2000 flies.

+ indicates a difference from the control at the $p<0.01$ level.

of the other lines (notably $\mathrm{L} S b \times G l 2$ and $\mathrm{L} S b \times$ Ser 1$)$ differed in the expected direction. Retests of these three selection lines were therefore carried out two months after the completion of the tests in table 2, together with the appropriate controls. Since no coupling versus repulsion differences were detected in the previous experiments, the technically simpler repulsion crosses were employed. Eight replicate bottle cultures were set up for each line, and for the $S b \times G l$ and $S b \times S e r$ controls, so that recombination frequencies based on about 5000 flies could be obtained for each cross. The mean recombination frequencies were as follows: $0.204 \pm 0.006(\mathrm{H} \mathrm{Sb} \times G l 1), 0.115 \pm$ $0.012(\mathrm{~L} \mathrm{Sb} \times G l 2), 0 \cdot 126 \pm 0.012$ (Control $S b \times$ $G l), 0.321 \pm 0.009$ (L $S b \times S e r ~ 1)$, and $0.348 \pm 0.009$ (Control $S b \times S e r)$. The only line to differ significantly from the control is again $\mathrm{H} \mathrm{Sb} \times G l 1$ $(\mathrm{p}<0.001)$.

\section{Significance of the results}

The evidence presented above shows that the only line to show a clear-cut response to selection was H $S b \times G l 1$, which from now on will simply be referred to as the $\mathrm{H}$ line. It is possible that some of the other lines had indeed responded to selection, but clearly any such responses would be too small to be useful for further genetic study, however the 1.6-fold increase exhibited by the $\mathrm{H}$ line was clearly potentially usable, and formed the material for all subsequent work.

It is at first sight odd that the recombination frequencies of several of the L lines, shown in table 1, should apparently demonstrate a response to selection (notably L $S b \times \operatorname{Ser} 2$ ), which is not visible in table 2 or in the later tests. Almost certainly, these apparent responses are due to the establishment of closely-linked recessive lethals associated with one of the marker genes. Consider, for example, the cross

$$
\frac{G l l+}{++S b} \times \frac{G l l+}{++S b}
$$

where $l$ indicates a lethal closely linked to the locus of $S b$. The $\mathrm{Gl}^{+} \mathrm{Sb}^{+}$recombinant chromosomes produced by the female would usually carry the lethal, and would be colethal with the $G l S b^{+}$ chromosomes from the male, producing a deficit in the frequency of the $G \mathrm{~S} S b^{+}$recombinant class. Examination of the frequencies of the individual genotypes in the selection line records showed such a decline in one of the recombinant classes in several cases. Kidwell $(1972 b)$ has reported the appearance of lethals in association with dominant markers in her selection lines for reduced $G l-S b$ recombination.

This hypothesis was tested further by extracting several wild-type chromosomes from each of the suspect selection lines (L Sb $\times$ Ser 2 and L Ser $\times$ $S b 1$ and 2). Both $\mathrm{L} S b \times \operatorname{Ser} 2$ and $\mathrm{L} \mathrm{Ser} \times S b 1$ proved to contain lethals at high frequencies; these were allelic within lines, but non-allelic between lines. Surprisingly, no lethals were found in 22 chromosomes from $\mathrm{L} S \mathrm{Se} \times \mathrm{Sb} 2$ despite evidence for a deficiency of $+S b$ chromosomes within this line. It is possible that their frequency had declined during the $2 \frac{1}{2}$ months of relaxed selection that preceded the lethal tests.

\section{Possible age effects}

One further possible artefact should be mentioned. There is evidence that the frequency of crossing 
over in chromosome 3 declines with age, particularly for regions near the centromere (Bridges, 1927). Since the $G l-S b$ region includes the centromere, it is conceivable that the increased frequency of recombination found in the $\mathrm{H}$ line is due either to slower development of the $\mathrm{H} \mathrm{Gl}+/+$ $S b$ Fl females or to earlier egg-laying by them, so that recombination frequencies based on 8-day emergences are biased towards progeny from younger females in this stock. The former possibility is most unlikely, since the control and $\mathrm{HFl}$ virgins were always collected over the same 3-day period from cultures set up at the same time, and no obvious differences in emergence pattern were noted. The second possibility can be tested by examining the relationship between $G l-S b$ recombination frequency and day of emergence, for both $\mathrm{H}$ and control $(\mathrm{C})$ cultures. The results of such a study are plotted in fig. 2, where it can be

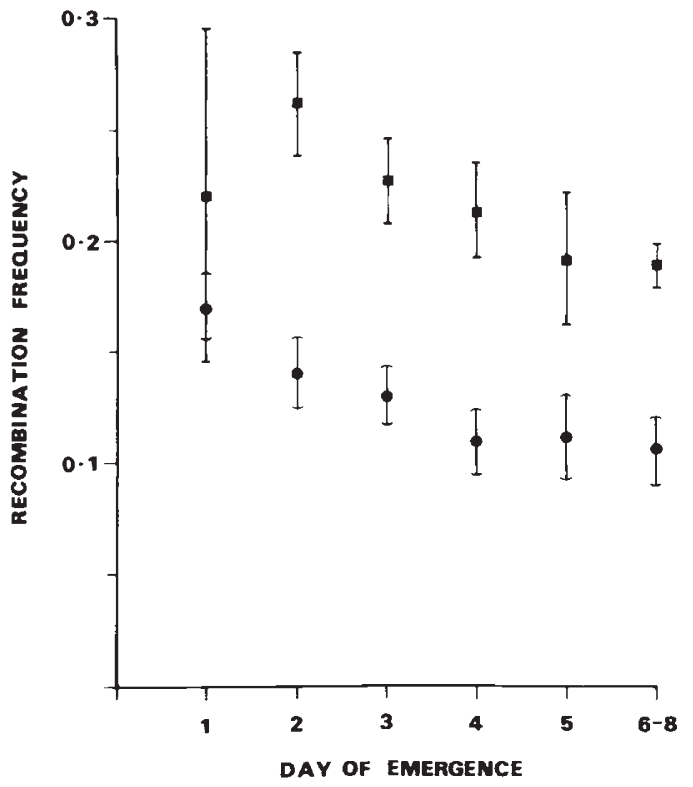

Figure 2 The $G l-S b$ recombination frequencies among progeny emerging on different days. The squares and circles represent the means of the $\mathrm{H}$ and $\mathrm{C}$ stocks, respectively; the bars give their 95 per cent confidence limits.

seen that the difference between $\mathrm{H}$ and $\mathrm{C}$ recombination frequencies is effectively independent of day of scoring. This is inconsistent with the second of the above possibilities. The fact that the early days of emergence of the $\mathrm{C}$ bottles have much lower recombination frequencies than the later days of emergence of the $\mathrm{H}$ bottles also excludes the first possibility.

\section{Effects of relaxed selection}

\section{Retests of the $\mathrm{H}$ stock}

As mentioned above, the $\mathrm{H}$ and $\mathrm{C}$ stocks were maintained by mass culture of the respective $G l$ and $S b$ flies, without any selection for increased recombination. It might be expected, therefore, that the recombination frequency between $G l$ and $S b$ would decline. Tests were carried out in July, 1980 and October 1981 (approximately 20 months and 35 months after the relaxation of selection). The first of these yielded a mean recombination frequency of $0 \cdot 181 \pm 0.004$, and the second a mean of $0.114 \pm 0.008$; these values may be compared with the values of $0 \cdot 224 \pm 0 \cdot 008$ and $0 \cdot 144 \pm 0 \cdot 006$ for the isogenic $\mathrm{H}$ stock described below, measured at approximately the same time. (The isogenic $\mathrm{C}$ stock gave values of $0.134 \pm 0.008$ and $0.086 \pm$ 0.006 respectively). There is therefore evidence for a decline in the recombination frequency of the non-isogenic $\mathrm{H}$ stock, although it still remains higher than the $\mathrm{C}$ stock.

The genetic basis of this was investigated in autumn 1982 by isolating individual $\mathrm{Gl}$ chromosomes from the mass-culture $\mathrm{H} G l$ stock, and substituting them into a background of isogenic $\mathrm{X}$ and 2nd chromosomes from the isogenic H stock (see below). The recombination frequencies of females heterozygous for each of these chromosomes were determined in vial cultures, on a background heterozygous for the $\mathrm{H}$ 2nd chromosome and the balancer $S M 1$ (which increases the fertility of the females). The recombination frequencies for four of these chromosomes were $0 \cdot 186 \pm 0 \cdot 010,0 \cdot 192 \pm$ $0 \cdot 016,0 \cdot 202 \pm 0 \cdot 016$ and $0 \cdot 195 \pm 0 \cdot 015$, which do not differ significantly. The overall mean is $0 \cdot 194 \pm$ $0 \cdot 007$. This compares with values of $0 \cdot 26$ and $0 \cdot 16$ yielded by extensive measurements of the $\mathrm{H}$ and $\mathrm{C}$ isogenic stocks, and indicates that an important element in the decline in recombination frequency of the mass-culture H stock (but probably not all) can be attributed to chromosome 3 .

\section{Resumption of selection}

Selection for increased $G l-S b$ recombination was resumed in July, 1980, using the same design as previously, and employing the mass-cultured $\mathrm{H} \mathrm{Gl}$ and $S b$ stocks as base material. The progress of the two replicate selection lines is shown in fig. 3 . After 10 generations, it was plain that no progress was being made, and the lines were discarded. The regression of recombination frequency on generation was (non-significantly) negative in both cases. The recombination frequency averaged over 


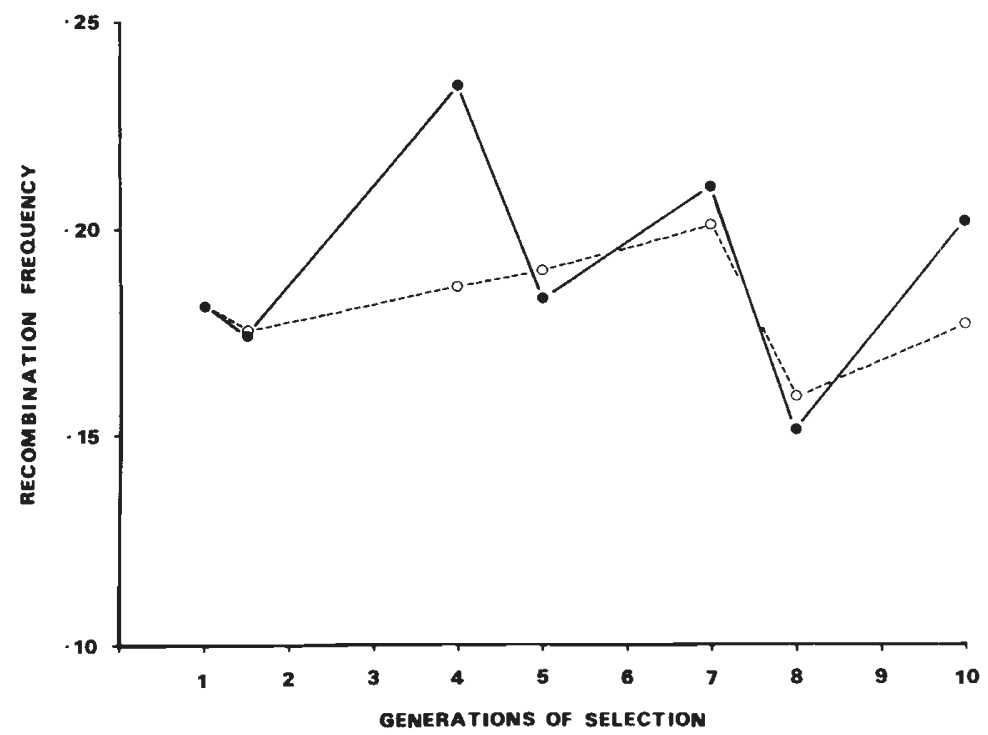

Figure 3 The responses to selection for increased $G I-S b$ recombination frequency in two replicate lines derived from the mass-cultured high recombination lines.

all generations was $0 \cdot 186 \pm 0 \cdot 004$, confirming the reduction in recombination frequency below the value for the isogenic $\mathrm{H}$ stock.

\section{ANALYSES OF THE H LINE}

From the above results, it can be concluded that the increased frequency of $G l-S b$ recombination observed for the $\mathrm{H} S b \times G l 1$ line in table 2 (the $\mathrm{H}$ stock) is a genuine genetic response to selection for increased recombination. Several different experiments were carried out to try and analyse the genetic basis of this response at the level of whole-chromosome, rather than individual gene, effects.

\section{Dominance tests}

The first step was to carry out measurements of the recombination frequencies of the reciprocal $F 1$ crosses between the $\mathrm{H}$ and $\mathrm{C}$ stocks. 8 replicate bottles of IV males mated to repulsion $G l+/+S b$ females from the $\mathrm{H}, \mathrm{C}, q \mathrm{H} \times \mathrm{C}$ o and $q \mathrm{C} \times \mathrm{H} \delta$ crosses were assayed ( 4 bottles of each cross were from $q G l \times S b$, and 4 were from $q S b \times G l \delta$ ). The mean recombination frequencies (based on about 5000 flies for each) were

$$
\begin{array}{lll}
\mathrm{H}: & 0.224 \pm 0.008 & \text { q } \mathrm{H} \times \mathrm{C} \delta: 0.178 \pm 0.006 \\
\mathrm{C}: & 0.134 \pm 0.008 & \text { q } \mathrm{C} \times \mathrm{H} \delta: 0 \cdot 165 \pm 0.005
\end{array}
$$

(No significant differences between $q G l \times S b$ ๘ and $q S b \times G l$ s were detected.)
The F1 is therefore approximately halfway between the parental lines, indicating an absence of directional dominance. The reciprocal difference is non-significant in these data, but later experiments showed it to be real.

\section{Male backcross experiment}

A preliminary study of the effects of individual chromosomes on recombination was carried out using a modification of the experimental design of Turner (1979) for analysis of a difference in recombination between two silkworm stocks. This technique exploits the absence of crossing over in males to carry out partial substitution of chromosomes between the $\mathrm{H}$ and $\mathrm{C}$ lines, as shown in table 3. Only the effects of chromosomes 1 and 3 can be detected directly within each backcross experiment, since chromosomes 2 and 4 are unmarked; in order to test for such effects, measurements of the $\mathrm{H}, \mathrm{C}$ and both reciprocal $\mathrm{Fl}$ crosses were made simultaneously with measurements of the female progeny of the backcrosses. The experiment was carried out in two replicate blocks, scored in separate weeks in September, 1979. Within each block, 3 or 4 bottle matings of 8 repulsion females with IV males were scored for each category of backcross female shown in table 3 , and for the line and reciprocal $F 1$ crosses. In several cases, (the Fl's and backcrosses 2, 4, 5 and 7), females can be distinguished according to whether they carry $G l$ or $S b$ H chromosomes; there was no significant difference overall between these 
Table 3 Design of the male backcross experiment

$0_{11}$ or $0_{C}$. denotes cytoplasm derived from the $\mathrm{H}$ or $\mathrm{C}$ lines respectively

$\mathrm{l}_{\mathrm{H}}$ or $\mathrm{l}_{\mathrm{C}}$ denotes chromosome $\mathrm{l}$ derived from the $\mathrm{H}$ or $\mathrm{C}$ lines respectively

$2_{\mathrm{H}}$ or $2_{\mathrm{C}}$ denotes chromosome 2 derived from the $\mathrm{H}$ or $\mathrm{C}$ lines respectively

$3_{\mathrm{H}}$ or $3_{\mathrm{C}}$ denotes chromosome 3 derived from the $\mathrm{H}$ or $\mathrm{C}$ lines respectively

Chromosome 4 behaves in the same way as 2 and is not indicated

Classes of $F_{1}$ male:

(a) $0_{\mathrm{H}} 1_{\mathrm{H}} \frac{2_{\mathrm{H}}}{2_{\mathrm{C}}} \frac{\mathrm{Sb}_{\mathrm{H}}}{\mathrm{Gl}_{\mathrm{C}}}$ from $\odot \mathrm{Sb}_{\mathrm{H}} \times \delta \mathrm{Gl}_{\mathrm{C}}$

(b) $0_{\mathrm{H}} 1_{\mathrm{H}} \frac{2_{\mathrm{H}}}{2_{\mathrm{C}}} \frac{\mathrm{Sb}_{\mathrm{C}}}{\mathrm{Gl} l_{\mathrm{H}}}$ from $\odot \mathrm{Gl}_{\mathrm{H}} \times ð \mathrm{Sb}_{\mathrm{C}}$

(c) $0_{C} \cdot l_{C} \frac{2_{\mathrm{H}}}{2} \frac{\mathrm{Sb}_{\mathrm{H}}}{\mathrm{Gl}_{\mathrm{C}}}$ from $q \mathrm{Gl}_{\mathrm{C}} \times \delta \mathrm{Sb}_{\mathrm{H}}$

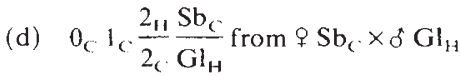

These are backcrossed to $\mathrm{H}$ and $\mathrm{C}$ females, to give the following types of backcross females

H Backcrosses

(1) $0_{\mathrm{H}} \frac{1_{\mathrm{H}}}{1_{\mathrm{H}}} \frac{3_{\mathrm{H}}}{3_{\mathrm{H}}}$

(3) $0_{H} \frac{l_{C}}{1_{H}} \frac{3_{H}}{3_{H}}$
C Backcrosses
(5) $0, \frac{1_{\mathrm{C}}}{\mathrm{I}_{\mathrm{H}}} \frac{3_{\mathrm{C}}}{3_{\mathrm{H}}}$
(6) $0_{C} \frac{1_{C}}{1_{\mathrm{H} 1}} \frac{3 \mathrm{C}}{3_{\mathrm{C}}}$
(7) $\quad 0<\frac{l_{C}}{1_{C} \cdot \frac{3_{C}}{3_{H}}}$
(8) $\quad 0, \frac{1,}{1_{C}} \frac{3 \mathrm{C}}{3 \mathrm{C}}$

In the $\mathrm{H}$ backcrosses, $\frac{1}{2}$ the females in each class are expected to be $2_{\mathrm{H}} / 2_{\mathrm{C}}$ and $\frac{1}{2}$ are $22_{\mathrm{H}} / 2_{\mathrm{H}}$. In the $\mathrm{C}$ backcrosses, the corresponding expectations are $\frac{1}{2} 2_{\mathrm{H}} / 2_{\mathrm{C}}$ and $\frac{1}{2} 2 \mathrm{C} / 2_{\mathrm{C}}$

types of female (the mean differences in recombination frequency between $G l_{\mathrm{H}}$ and $S b_{\mathrm{H}}$ females being -0.002 and -0.001 in blocks 1 and 2 respectively). This is in agreement with the Fl results above, and $G l_{\mathrm{H}}$ and $S b_{\mathrm{H}}$ genotypes were accordingly pooled in the following analysis.

For the purpose of statistical tests, the recombination frequencies were transformed to angles (the untransformed means for each category of female are shown in table 4). Estimates of the error variance for each block were obtained by pooling the error sums of squares for the backcross, line and Fl sums of squares, and these were used in $t$-tests for the significance of the genetic effects. Poor culture conditions in the second block gave a significantly higher error variance than for the first block, making a pooled analysis of variance impossible $\left(s^{2}=0.000942\right.$ and 0.00195 respectively; $\left.F_{36,34}=2 \cdot 1, \quad p<0.05\right)$. The significance values of the $t$-tests for the individual effects in blocks 1 and 2 were combined by Fisher's $\chi^{2}$ method to yield the significance levels shown in table 4 . The chromosome effects averaged over both blocks are also shown in table 4; these are calculated from the appropriate differences in means e.g., the effect $l_{H} / l_{H}$ vs. $l_{H} / l_{C}$ is calculated as the difference between the means of females of types 1 and 2 on the one hand, and 3 and 4 on the other; the effect $2_{\mathrm{H}} / 2_{\mathrm{H}}$ vs. $2_{\mathrm{H}} / 2_{\mathrm{C}}$ is calculated as twice the difference between the means of $\mathrm{H}$ and type 4 females, and the means of type 1 and $\mathrm{H} \times \mathrm{C}$ females, etc.

The $\mathrm{X}$ chromosome effect only borders on significance in the contrast $l_{H} / l_{C}$ vs. $l_{C} / l_{C}$ (this effect has $\mathrm{p}<0.05$ in block $\mathrm{l}$, but is non-significant in block 2). The contrast $2_{\mathrm{H}} / 2_{\mathrm{H}}$ vs. $2_{\mathrm{H}} / 2_{\mathrm{C}}$ is significantly positive, whereas the contrast $2_{\mathrm{H}} / 2_{\mathrm{C}}$ vs. $2_{\mathrm{C}} / 2_{\mathrm{C}}$ is negative, but not significantly so. The $3 \mathrm{rd}$ chromosome effect $3_{\mathrm{H}} / 3_{\mathrm{C}}$ vs. $3_{\mathrm{C}} / 3_{\mathrm{C}}$ is significantly positive. The reciprocal difference $\mathrm{H} \times \mathrm{C}$ vs. $\mathrm{C} \times \mathrm{H}$ is significant when its probability value is pooled with that from the experiment described previously in Dominance tests. The overall difference between the $\mathrm{H}$ and $\mathrm{C}$ lines in this experiment is 0.11 ; if the chromosomal effects are taken at face value, their joint total effect $(0.010+0.021+0.044=0.075)$, together with the reciprocal effect of 0.026 , account for most of the overall difference. This conclusion should not be taken very seriously, however, as there is evidence from later experiments for interactions between chromosomes (Charlesworth and Charlesworth, 1985). 
Table 4 Results of the male backcross experiments

The mean recombination frequency and its standard error (below the mean) are shown for each category of female.

Block 1

H Backcross

(1) 0.181

0.014

(3) 0.185

0.010
(2) 0.156

0.014

(4) $0 \cdot 152$

0.006
C Backcross
(5) 0.130
0.005
(7) 0.126
0.010
(6) $\begin{array}{r}0.134 \\ 0.012 \\ \text { (8) } 0.098 \\ 0.007\end{array}$

Lines and $F 1$ 's

$\begin{array}{llll}\mathrm{H} & 0.192 & \mathrm{H} \times \mathrm{C} & 0.142 \\ & 0.015 & & 0.012 \\ \mathrm{C} & 0.090 & \mathrm{C} \times \mathrm{H} & 0.106 \\ & 0.007 & & 0.009\end{array}$

Block 2
(6) $0 \cdot 118$
0.022
(8) 0.102
0.008
(1) $\begin{array}{r}0.178 \\ 0.026\end{array}$
(2) $0 \cdot 185$
0.020
(5) $\begin{aligned} & 0.151 \\ & 0.009\end{aligned}$
(7) 0.137
0.014
0.007
H $\quad 0.230$
0.017
C 0.110
$\mathrm{H} \times \mathrm{C} \quad 0 \cdot 142$
$0 \cdot 024$
$\mathrm{C} \times \mathrm{H} \quad 0 \cdot 125$
$0 \cdot 002$

The genetic effects, estimated from all the available data, are as follows

$\begin{array}{lcc}\text { Chromosome 1: } & 1_{\mathrm{H}} / 1_{\mathrm{H}} \text { vs. } 1_{\mathrm{H}} / 1_{\mathrm{C}} & 1_{\mathrm{H}} / 1_{\mathrm{C}} \text { vs. } 1_{\mathrm{C}} / 1_{\mathrm{C}} \\ & -0.008 & 0.018 \\ \text { Chromosome 2: } & 2_{\mathrm{H}} / 2_{\mathrm{H}} \text { vs. } 2_{\mathrm{H}} / 2_{\mathrm{C}} & 2_{\mathrm{H}} / 2_{\mathrm{C}} \text { vs. } 2_{\mathrm{C}} / 2_{\mathrm{C}} \\ & 0.056^{*} & -0.025 \\ \text { Chromosome 3: } & 3_{\mathrm{H}} / 3_{\mathrm{H}} \text { vs. } 3_{\mathrm{H}} / 3_{\mathrm{C}} & 3_{\mathrm{H}} / 3_{\mathrm{C}} \text { vs. } 3_{\mathrm{C}} / 3_{\mathrm{C}} \\ & 0.021 & 0.023^{*}\end{array}$

Reciprocal effect: $\quad \mathrm{H} \times \mathrm{C}$ vs. $\mathrm{C} \times \mathrm{H}$

$0.026^{*}$

\section{Construction of isogenic stocks}

The above experiments were carried out using $G l$ and $S b$ chromosomes maintained in mass culture without selection for recombination. The $\mathrm{H}$ stocks eventually showed a decline in recombination frequency. In order to guard against such a loss of response, and to avoid complicating further genetic analyses by genetic variation within $\mathrm{H}$ and $\mathrm{C}$ stocks, isogenic $\mathrm{H}$ and $\mathrm{C}$ stocks were constructed during 1979, and formed the basis for all further work. These stocks were constructed using the balancer chromosomes described in the second section of "Materials and Methods". The breeding programmes, although laborious, involved no new principles and details will not be given here.

The first step was to construct stocks homozygous for single $\mathrm{X}$ chromosomes derived from the $\mathrm{H}$ and $\mathrm{C}$ lines. This was done by crossing females homozygous for FM7 to single males, allowing the $\mathrm{F} 1 F M 7 /$ + females and males to interbreed, and mating together the resulting $F M 7 /+$ females and the + males. In this way, sets of 5 lines homozygous for independently extracted chromosomes from each of the $\mathrm{H}$ and $\mathrm{C} G l$ and $S b$ stocks were obtained. The second step was to isolate pairs of 2nd and 3rd chromosomes from each of the $\mathrm{H}$ and $\mathrm{C}$ stocks. The $3 \mathrm{rd}$ chromosomes were either $\mathrm{Gl}$ or
$S b$, and were balanced over TM6. From among these lines, some were chosen that were reasonably viable and fertile when homozygous for the 2nd chromosome. Second chromosomes were then substituted between pairs of $\mathrm{HGl}$ and $\mathrm{H} \mathrm{Sb}$ and between pairs of $\mathrm{C} G l$ and $\mathrm{C} S b$ lines, in such a way that a number of pairs of lines were obtained that shared a 2nd chromosome, but differed with respect to having $G l$ or $S b 3$ rd chromosomes. The final step was to transfer these combinations of 2nd and 3rd chromosomes onto a background isogenic for one of the extracted X chromosomes. The cytoplasm in each case was derived from the original FM7 stock.

Two pairs of lines were chosen from among these lines for use in further investigations, on the basis of the following criteria.

(a) Each line had the same combination of 1 st and 2 nd chromosomes derived either from the $\mathrm{C}$ or from the $\mathrm{H}$ stocks, but differed in having $G l(\mathrm{C}$ or $\mathrm{H})$ versus $S b(\mathrm{C}$ or $\mathrm{H})$. In what follows, $\mathrm{C} \mathrm{C} G l_{\mathrm{C}}$, $\mathrm{CC} S b_{\mathrm{C}}, \mathrm{H} \mathrm{H} G l_{\mathrm{H}}$ and $\mathrm{HH} S b_{\mathrm{H}}$ will be used to denote the chromosomal composition of these 4 stocks, with each letter symbolising the state of the appropriate chromosome (1, 2 or 3 in order). Note that the $\mathrm{X}$ and 2nd chromosomes are identical within the $\mathrm{C}$ pair and within the $\mathrm{H}$ pair, whereas 
the $G l$ and $S b$ chromosomes with the same subscript may differ at many loci.

(b) No salivary chromosome rearrangements were detected among 5 female larvae of each Fl between line males and $c n b w$; rie.

(c) The recombination rates in $G l+/+S b$ females formed by intercrossing the two $\mathrm{C}$-derived lines, and the two $\mathrm{H}$-derived lines, were similar to those for the original $\mathrm{C}$ and $\mathrm{H}$ stocks.

(d) There was no evidence for secondary nondisjunction due to the presence of XXY females, in matings between females from the 4 stocks and $F M 7$ males. It was necessary to check this because of the elevated rate of primary non-disjunction in females heterozygous for $\mathrm{X}$-chromosome inversions (Sturtevant and Beadle, 1936). If this had occurred, crosses between the isogenic $\mathrm{H}$ and $\mathrm{C}$ stocks might have given misleading results as far as the $\mathrm{X}$ chromosome was concerned. Out of 7391 progeny tested from the 4 stocks, only 2 nondisjunction individuals were found, which is well within the limits expected for normal primary nondisjunction rates.

\section{Genetic analyses of the isogenic stocks}

\section{Values of the lines and FI's}

The first experiment was to carry out simultaneous measurements of the $G l-S b$ recombination frequency in the $\mathrm{H}$ and $\mathrm{C}$ isogenic stocks and in the reciprocal Fl's between them. The means and standard errors were as follows:

$$
\begin{aligned}
\mathrm{H}: & 0 \cdot 222 \pm 0.0193 \\
S b_{\mathrm{H}} \times G l_{\odot}: & 0 \cdot 156 \pm 0.006 \\
G l_{\mathrm{H}} \times S b_{\mathrm{C}}: & 0 \cdot 190 \pm 0 \cdot 012 \\
\mathrm{C}: & 0 \cdot 143 \pm 0 \cdot 010 \\
G l_{\mathrm{C}} \times S b_{\mathrm{H}}: & 0 \cdot 128 \pm 0 \cdot 005 \\
S b_{\mathrm{C}} \times G l_{\mathrm{H}}: & 0 \cdot 178 \pm 0.010
\end{aligned}
$$

(The $\mathrm{H}$ and $\mathrm{C}$ means are based on the means for the reciprocal crosses $G l_{\mathrm{H}} \times S b_{\mathrm{H}}, S b_{\mathrm{H}} \times G l_{\mathrm{H}}$, and $G l_{C} \times S b_{C}, \quad S b_{C} \times G l_{C} \quad$ respectively, since no reciprocal differences were observed in these crosses).

The difference between the isogenic $\mathrm{H}$ and $\mathrm{C}$ stocks is, within the limits of sampling error, similar to that detected earlier, and is highly significant on a $t$-test of the angular transforms. The reciprocal difference between the two types of $S b_{\mathrm{H}} \times G l_{\mathrm{C}} \quad \mathrm{F} 1$ is highly significant $\left(t_{8}=3 \cdot 35, \mathrm{p}<\right.$ $0 \cdot 01)$; the $S b_{C} \times G l_{\mathrm{H}}$ reciprocal difference is nonsignificant with these data, but was found to be significant in a replicate experiment $\left(t_{11}=2 \cdot 21\right.$, $\mathrm{p}<0.025)$. A curious feature of the $\mathrm{Fl}$ data is that the $G l_{\mathrm{H}} \times S b_{C}$ crosses have higher values than the $S b_{\mathrm{H}} \times G l_{C} \quad$ crosses; averaging over the two reciprocal crosses, this difference amounts to 0.042 recombination units, and is significant at the 1 per cent level. This suggests that the $G l_{\mathrm{H}} 3 \mathrm{rd}$ chromosome has a larger dominant effect in increasing recombination than the $S b_{\mathrm{H}} 3$ rd chromosome, an interpretation that is confirmed by later experiments (Charlesworth and Charlesworth, 1985).

\section{Female backcross experiments}

The genetic basis of the reciprocal effect was investigated by means of a series of backcross experiments involving the isogenic $\mathrm{C}$ and $\mathrm{H}$ stocks. These experiments were designed to produce females with similar nuclear genotypes but differing in whether their cytoplasm was derived from the $\mathrm{C}$ or $\mathrm{H}$ stocks. Three generations of backcrossing of females to the $\mathrm{C}$ and $\mathrm{H}$ isogenic stocks were carried out. The 1 st and 2 nd chromosomes were exposed to free recombination, but recombination in chromosome 3 was prevented by using females with TM6 heterozygous over $\mathrm{H}$ or $\mathrm{C}$ chromosomes for the backcross. The expected genotypes of females from the various backcross generations whose recombination fractions were assayed are shown in table 5 , where the states of the lst and 2 nd chromosomes are represented as if no crossing over were occurring in females. The first backcross generations were set up in three separate blocks (corresponding to different weeks), all eight classes of female being assayed in several replicate bottle cultures in each block. The second and third backcrosses were assayed in a similar way, but only two blocks were set up. The data were transformed to angles, and analysed by means of analysis of variance and $t$-tests of differences in means. A summary of the data, expressed as the means for each class of female in each backcross generation over all replicates and blocks, is given in table 6 .

The genetic basis of the reciprocal effect can be examined by comparing the mean for genotypes 1-4 (which possess the cytoplasm of the C stock) with the mean for genotypes 5-8 (which possess the cytoplasm of the $\mathrm{H}$ stock). If the reciprocal effect were due to a cytoplasmic factor, it should persist in all three backcross generations. If it were due to a maternal effect of the nuclear genotype, it should be non-existent in all three generations, since there is no difference in maternal nuclear genotypes between the pairs 1 and 5,2 and 6, etc. It can be seen from table 6 that the cytoplasmic effect in backcross 1 females is comparable in value 
Table 5 Genotypes of females assayed for Gl-Sb recombination, from the 3 backcross generations

\begin{tabular}{|c|c|c|c|}
\hline Genotype No. & $\begin{array}{l}\text { 1st \& 2nd } \\
\text { Chromosome genotypes }\end{array}$ & $\begin{array}{l}\text { 3rd } \\
\text { Chromosom }\end{array}$ & notypes \\
\hline Backcross 1 & & Own & Maternal \\
\hline $\begin{array}{l}1 \& 5 \\
2 \& 6 \\
3 \& 7 \\
4 \& 8\end{array}$ & $\begin{array}{l}\frac{1}{2} \mathrm{H} / \mathrm{C}: \frac{1}{2} \mathrm{C} / \mathrm{C} \\
\frac{1}{2} \mathrm{H} / \mathrm{C}: \frac{1}{2} \mathrm{H} / \mathrm{H} \\
\frac{1}{2} \mathrm{H} / \mathrm{C}: \frac{1}{2} \mathrm{C} / \mathrm{C} \\
\frac{1}{2} \mathrm{H} / \mathrm{C}: \frac{1}{2} \mathrm{H} / \mathrm{H}\end{array}$ & $\begin{array}{l}S b_{\mathrm{C}} / G l_{\mathrm{C}} \\
G l_{\mathrm{H}} / S b_{\mathrm{C}} \\
G l_{\mathrm{H}} / S b_{\mathrm{C}} \\
G l_{\mathrm{H}} / S b_{\mathrm{H}}\end{array}$ & $\begin{array}{l}T M 6 / S b_{\mathrm{C}} \\
T M 6 / S b_{\mathrm{C}} \\
T M 6 / G l_{\mathrm{H}} \\
T M 6 / G l_{\mathrm{H}}\end{array}$ \\
\hline
\end{tabular}

The backcross 1 females were derived by crossing $T M 6 / S b$ or $T M 6 / G l$ females from the Fl's between $\mathrm{H} \mathrm{H} \mathrm{Gl}_{\mathrm{H}}$ and C C $S b_{\mathrm{C}}$ to $\mathrm{Gl}$ or $S b$ males, respectively, from the $\mathrm{H}$ and $\mathrm{C}$ isogenic stocks. Genotypes $1-4$ were derived from $\mathrm{Fl}$ females from the cross $q \mathrm{C} \times \mathrm{H} \delta$, and genotypes $5-8$ from the cross $q \mathrm{H} \times \mathrm{C} \delta$

Backcross 2

$\begin{array}{llll}1 \& 5 & \frac{1}{4} \mathrm{H} / \mathrm{C}: \frac{3}{4} \mathrm{C} / \mathrm{C} & S b_{\mathrm{C}} / G l_{\mathrm{C}} & T M 6 / G l_{\mathrm{C}} \\ 2 \& 6 & \frac{1}{4} \mathrm{H} / \mathrm{C}: \frac{3}{4} \mathrm{H} / \mathrm{H} & G l_{\mathrm{H}} / S b_{\mathrm{H}} & T M 6 / G l_{\mathrm{H}} \\ 3 \& 7 & \frac{1}{4} \mathrm{H} / \mathrm{C}: \frac{3}{4} \mathrm{C} / \mathrm{C} & S b_{\mathrm{C}} / G l_{\mathrm{C}} & T M 6 / S b_{\mathrm{C}} \\ 4 \& 8 & \frac{1}{4} \mathrm{H} / \mathrm{C}: \frac{3}{4} \mathrm{H} / \mathrm{H} & G l_{\mathrm{H}} / S b_{\mathrm{H}} & T M 6 / S b_{\mathrm{H}}\end{array}$

Backcross 3

$1 \& 5$

$2 \& 6$

$3 \& 7$

$4 \& 8$

$$
\begin{aligned}
& \frac{1}{8} \mathrm{H} / \mathrm{C}: \frac{7}{8} \mathrm{C} / \mathrm{C} \\
& \frac{1}{8} \mathrm{H} / \mathrm{C}: \frac{7}{8} \mathrm{H} / \mathrm{H} \\
& \frac{1}{8} \mathrm{H} / \mathrm{C}: \frac{7}{8} \mathrm{C} / \mathrm{C} \\
& \frac{1}{8} \mathrm{H} / \mathrm{C}: \frac{7}{8} \mathrm{H} / \mathrm{H}
\end{aligned}
$$

$\begin{array}{ll}S b_{\mathrm{C}} / G l_{\mathrm{C}} & T M 6 / G l_{\mathrm{C}} \\ G l_{\mathrm{H}} / S b_{\mathrm{H}} & T M 6 / G l_{\mathrm{H}} \\ S b_{\mathrm{C}} / G l_{\mathrm{C}} & T M 6 / S b_{\mathrm{C}} \\ G l_{\mathrm{H}} / S b_{\mathrm{H}} & T M 6 / G l_{\mathrm{H}}\end{array}$

Backcross 2 and Backcross 3 females of a given number $(1-8)$ were derived by crosses of $T M 6 / S b$ or $T M 6 / G l$ females, from backcross generations 1 and 2 respectively, to

\begin{tabular}{|c|c|c|c|c|c|}
\hline Genotype & $\begin{array}{l}\text { Mean rec. } \\
\text { frequency }\end{array}$ & $\begin{array}{l}\text { No. of } \\
\text { cultures }\end{array}$ & Genotype & $\begin{array}{l}\text { Mean rec. } \\
\text { frequency }\end{array}$ & $\begin{array}{l}\text { No. of } \\
\text { cultures }\end{array}$ \\
\hline \multicolumn{6}{|c|}{ Backcross 1} \\
\hline 1 & $0 \cdot 128 \pm 0 \cdot 005$ & 10 & 5 & $0.138 \pm 0.005$ & 11 \\
\hline 2 & $0 \cdot 170 \pm 0.006$ & 9 & 6 & $0 \cdot 189 \pm 0 \cdot 007$ & 8 \\
\hline 3 & $0.145 \pm 0.005$ & 13 & 7 & $0 \cdot 153 \pm 0 \cdot 005$ & 14 \\
\hline 4 & $0 \cdot 178 \pm 0 \cdot 005$ & 15 & 8 & $0 \cdot 199 \pm 0 \cdot 006$ & 13 \\
\hline \multicolumn{6}{|c|}{ Cytoplasmic effect is 0.014} \\
\hline \multicolumn{6}{|c|}{ Backcross 2} \\
\hline 1 & $0 \cdot 106 \pm 0.004$ & 8 & 5 & $0.112 \pm 0.007$ & 8 \\
\hline 2 & $0.178 \pm 0.008$ & 8 & 6 & $0 \cdot 169 \pm 0.008$ & 8 \\
\hline 3 & $0 \cdot 121 \pm 0.007$ & 8 & 7 & $0 \cdot 119 \pm 0 \cdot 009$ & 5 \\
\hline 4 & $0 \cdot 181 \pm 0 \cdot 013$ & 3 & 8 & $0 \cdot 186 \pm 0 \cdot 016$ & 2 \\
\hline \multicolumn{6}{|c|}{ Cytoplasmic effect is 0.000} \\
\hline \multicolumn{6}{|c|}{ Backcross 3} \\
\hline 1 & $0.115 \pm 0.005$ & 12 & 5 & $0 \cdot 116 \pm 0 \cdot 006$ & 8 \\
\hline 2 & $0 \cdot 180 \pm 0.007$ & 8 & 6 & $0 \cdot 196 \pm 0.008$ & 7 \\
\hline 3 & $0.118 \pm 0.009$ & 4 & 7 & $0 \cdot 124 \pm 0.006$ & 8 \\
\hline 4 & $0 \cdot 169 \pm 0.01 \mathrm{I}$ & 3 & 8 & $0.135 \pm 0.013$ & 2 \\
\hline \multicolumn{6}{|c|}{ Cytoplasmic effect is -0.003} \\
\hline
\end{tabular}
males from the $\mathrm{H}$ and $\mathrm{C}$ isogenic stocks

Table 6 Summary of measurements of $G l-S b$ recombination frequencies in females of each backcross generation 
to that found previously, and is highly significant $\left(t_{69}=3.55, \mathrm{p}<0.001\right)$. There is no evidence for a significant effect in generations 2 and 3 . This suggests strongly that the reciprocal effect is not due to a purely cytoplasmic factor, which is consistent with the fact that the isogenic $\mathrm{C}$ and $\mathrm{H}$ stocks used here have a common cytoplasm derived from the FM7 stock. Furthermore, it seems that the grandmaternal rather than the maternal genotype influences the recombination frequency, since the reciprocal effect persists in backcross 1 .

The data for backcross 2 shed some further light on this problem. As shown in table 5, the contrasts between the means of genotypes 4 and 8 with those of 2 and 6 , and between the means of 3 and 7 with 1 and 5, test the grandmaternal effect of the $G l_{\mathrm{H}} 3$ rd chromosome when heterozygous with TM6. The $t$-value for the pooled contrast is $1.93(p<0.025$ on a one-tailed test $)$, and the value of the contrast is 0.012 , which is comparable with the previous value. This suggests that the grandmaternal effect is due mainly to chromosome 3.

Backcross 1 also provides information on nuclear gene effects. Ignoring for the moment the effects of recombination, the contrast of the means of genotypes 2 and 6 with those of 3 and 7 provides a test of the effects of chromosomes 1 and 2 . The value of this contrast is $0.030(t=5.57, \mathrm{p}<0.001)$. It can also be seen that the most extreme backcross genotypes ( 1 and 8 ) are close to the values for the $\mathrm{C}$ and $\mathrm{H}$ stocks respectively. This suggests that only a small number of genes on chromosomes other than 3 can be involved; since later chromosome substitution experiments showed effects of both chromosomes 1 and 2 on recombination, this probably indicates the presence of a single major factor on each of these chromosomes. The detailed interpretation of this problem, taking into account interchromosomal interactions, is discussed in Charlesworth and Charlesworth (1985).

Backcross 1 also shows evidence for a highly significant effect of chromosome 3 . The contrast of the mean of genotypes 4 and 8 with that of 2 and $6(0.009, \mathrm{p}>0.05)$ estimates the difference between $S b_{\mathrm{H}}$ and $S b_{\mathrm{C}}$ when heterozygous with $G l_{\mathrm{H}}$. Similarly, the contrast of the means of 3 and 7 with 1 and 5 estimates the difference between $G l_{\mathrm{H}}$ and $G l_{C}$ on a background of $S b_{\mathrm{C}}(0.016, \mathrm{p}<0.01)$. These data suggest that $S b_{\mathrm{H}}$ has a smaller effect on recombination than $G l_{\mathrm{H}}$, which is consistent with the findings on the Fl's between the $\mathrm{H}$ and $\mathrm{C}$ isogenic stocks (section 4). This is confirmed by later experiments, which indicate a complete lack of genes for increased recombination on the $S b_{\mathrm{H}}$ chromosome (Charlesworth and Charlesworth, 1985).

\section{CONCLUSIONS}

These results will be discussed in conjunction with the results of more detailed genetic studies in the accompanying paper (Charlesworth and Charlesworth, 1985). It may, however, be helpful to summarise the main conclusions of this paper at this point.

(a) Selection for increased and decreased $\mathrm{Gl}$ $S b$ and $S b-S e r$ recombination was generally ineffective, with only one line out of sixteen (for increased $G l-S b$ recombination) showing a definite response.

(b) The genes responsible for the increased rate of $G l-S b$ recombination did not show dominance in the Fl with the unselected control stock. This contrasts with the results of Kidwell $(1972 a, b)$, who observed that low recombination was recessive to high in her studies of variation in $G l-S b$ recombination.

(c) There was a small $(\approx 1 \cdot 5$ per cent $)$ reciprocal difference in the $\mathrm{F} 1$, which was controlled by a grandmaternal effect of the nuclear genotype, probably involving chromosome 3 .

(d) There was clear evidence for effects of genes on chromosomes 2 and 3 , and less clear evidence for an effect of the $\mathrm{X}$ chromosome.

Acknowledgements We are grateful to Dr P. T. Ives for providing us with wild-caught flies, and to $\mathrm{DrC}$ C. H. Langley for his hospitality during our visit to his laboratory at NIEHS, North Carolina during 1977-78. We thank Joan West and Doris Williams for their technical assistance with this work, which was supported by an SERC grant (GR/A/1253.3), and by $\mathrm{NIH}$ visiting fellowships to B.C. and I).C. for $1977 / 8$.

\section{REFERENCES}

BAILEY, N. T. J. 1961. Introduction to the Mathematical Theory of Genetic Linkage. Oxford University Press, Oxford.

BAKER, B. S., CARPENTER, A. T. (.., ESPOSITO, M. S., ESPOSITO, R. E. AND SANDLER, L. 1976. The genetic control of meiosis. Ann. Rev. Genet., 10, 53-134.

BRIICBES, C B. 1927. The relation of the age of the female to crossing over in the third chromosome of Drosophila melanogaster. J. Gen. Physiol., 8, 698-700.

(ATCHLSIDE, 1). (i. 1977. The Genetics of Recombination. Arnold, London.

CHARLESWORTH, B. AND (:HARLESWORTH, 1) 1985. Genetic variation in recombination in Drosophila. II. Genetic analysis of a high recombination stock. Heredity, 54, 85-98.

CHINNICI, J. P. 197Ia. Modification of recombination in Drosophila. I. Selection for increased and decreased crossing over. Genetics, 69, 71-83. 
CHINNICI, J. P. $1971 \mathrm{~b}$. Modification of recombination in Drosophila. 11. The polygenic control of crossing over. Genetics, 69, 85-96.

CROW, J. F. AND kimura, M. 1970. An Introduction to Population Genetics Theory. Harper and Row, New York.

EBINUMA, H. AND YOSHITAKE, N. 1981. The genetic system controlling recombination in the silkworm. Genetics, 99, 231-245.

FLEXON, P. B. AND RODELL, C. F. 1982. Genetic recombination and directional selection for DDT resistance in Drosophila melanogaster. Nature, 298, 672-674.

KIDWELL, M. G. $1972 a$. Genetic change of recombination value in Drosophila melanogaster. I. Artificial selection for high and low recombination and some properties of recombination-modifying genes. Genetics, 70, 419-432.

KIDWELL, M. G. $1972 b$. Genetic change of recombination value in Drosophila melanogaster. II. Simulated natural selection. Genetics, 70, 433-443.

LINDSLEY, D. L. AND GRELL, E. H. 1968. Genetic Variations of Drosophila melanogaster. Carnegie Institute, Washington (Publ. 627).

LINDSLEY, D. L. AND SANDLER, L. 1977. The genetic analysis of meiosis in female Drosophila melanogaster. Phil. Trans. Roy. Soc. Lond. B., 227, 295-312.
LINDSLEY, D. L. AND ZIMM, G. 1982. Computerised stock list 3. Drosophila Inf. Service, 57, 1-212.

MAYNARD SMITH, J. 1978. The Evolution of Sex. Cambridge Univ. Press, Cambridge.

NiCOLETTI, B. 1959. An efficient method for salivary gland preparations. Drosophila Inf. Service, 33, 181-182.

ROSE, M. R. AND CHARLESWORTH, B. 1981. Genetics of life history in Drosophila melanogaster. I. Sib analysis of adult females. Genetics, 97, 173-186.

Stahl, F. W. 1979. Genetic Recombination. Thinking About It in Phage and Fungi. Freeman, San Francisco.

STURTEVANT, A. H. AND BEADLE, G. W. 1936. The relation of inversions in the $\mathrm{X}$-chromosome of Drosophila melanogaster to crossing over and disjunction. Genetics, 21, 554-604.

TURNER, J. R. G. 1979. Genetic control of recombination in the silkworm. 1. Multigenic control of chromosome 2. Heredity, 43, 273-293.

Whitehouse, H. L. K. 1982. Genetic Recombination. Understanding the Mechanisms. Wiley, Chichester. 